\title{
Comparison of Diesel Tractor Emissions in Korea
}

\author{
Gyu Gang Han ${ }^{1}$, Jun Hyuk Jeon ${ }^{2}$, Myoung Ho Kim ${ }^{2,3,4}$, Jeong Min Lee ${ }^{5}$ and Seong Min Kim 1,2,3,4* \\ 1 Department of Agricultural Convergence Technology, Graduate School, Jeonbuk National University, \\ Jeonju, 54896, Korea; dt200v@jbnu.ac.kr \\ 2 Department of Agriculture Machinery Engineering, Graduate School, Jeonbuk National University, Jeonju, \\ 54896, Korea; splinter9608@jbnu.ac.kr (J.H.J.); myoung59@jbnu.ac.kr (M.H.K.) \\ 3 Department of Bioindustrial Machinery Engineering, College of Agriculture and Life Sciences, Jeonbuk \\ National University, Jeonju, 54896, Korea; \\ 4 Institute for Agricultural Machinery \& ICT Convergence, Jeonbuk National University, Jeonju, 54896, Korea; \\ 5 National Institute of Agricultural Sciences, Rural Development Administration, Jeonju, 54875, Korea; \\ jmk526@korea.kr \\ * Correspondence: smkim@jbnu.ac.kr; Tel.: +82-63-270-2617
}

\begin{abstract}
Due to the shortage of agricultural labor forces and rapid aging of farmers, the utilization of tractors is becoming popular and essential in Korea. Tractors can be classified into two types, a walking tractor called as a power tiller and a riding tractor. In this study, agricultural tractors including walking and riding types were categorized into 4 levels by rated output power. And diesel emission inventory of tractors was established and analyzed using 2011 and 2019 survey data in Korea. Emission inventory including CO, NOx, SOx, TSP(PM10), $\mathrm{PM}_{2.5}$, VOCs and $\mathrm{NH}_{3}$ were established using Tier 3 methodology. The total amount of emission using agricultural tractors was decreased about 13\% from 2011 to 2019 . The number of walking tractors were decreased by about $19 \%$ in 8 years, on the other hand that of riding tractors were increased by about $12 \%$. However, the emission reduction is about $48 \%$ for walking tractors and the emission increment is about $5 \%$ for riding tractors. Thus, the total emission from agricultural tractors was decreased by about $16 \%$ in those periods. It is due to the decrease of $21 \%$ and $15 \%$ in the hours of use of walking and riding tractors, respectively, in 2019. Walking tractors mainly emit air pollutants from spraying and transporting. Riding tractors mainly $61 \%$ of total air pollutants emits from soil preparation and transporting operations. Geographic information system (GIS) was used to spatially assign air pollutants variables into 17 provinces and metropolitan cities in Korea. High emission generating regions and changes of emissions during 8 years were clearly seen in GIS analysis. High air pollutant emitting regions are mainly located in the western and southern regions of Korea, which have plenty of arable areas compared to other regions in Korea.
\end{abstract}

Keywords: Agricultural Tractor; Diesel Emission; Air Pollutants; Emission Inventory; Geographic Information System

\section{Introduction}

In 2019, the total cultivated land in Korean was 1,643,465 ha, and the amount of production was $4,375 \times 10^{3}$ tons, $8,186 \times 10^{3}$ tons, 2,206 $\times 10^{3}$ tons and $65 \times 10^{3}$ tons of grain crops, vegetables, fruits, and specialty crops, respectively $[1,2]$. Due to the decline of agricultural labor forces and rapid aging of farmers, the utilization of agricultural machinery is becoming popular. Recently, there is a trend that farmers are utilizing bigger size tractors comparing to 2010 in Korea [3]. The mechanization rate of rice farming is more than $99 \%$, and that of the other major agricultural works in open field farming is about $62 \%$ in average in 2019 [4].

Tractor is a multi-purpose vehicle that performs major agricultural operations while driving with various implements such as a plow, a rotary, or a baler, and is frequently used in Korean agriculture [5, 6]. Tractors are used to do many agricultural practices such as tillage, harrowing, fertilizer and compost spreading, and transportation in almost every 
agricultural sector of grain, vegetable, fruit production, and livestock husbandry. Tractors usually use a diesel as a fuel and emit a lot of pollutant substances which are primary and secondary sources of particulate matter (PM) and the other air pollutants. PM is considered as one of the most concerning air pollutants due to its effect on human health and environment both in urban and rural area [7, 8, 9, 10, 11, 12, 13]. The non-road mobile machinery sector including tractors largely contributes to the emissions of PM10 and PM2.5, being responsible for $7.4 \%$ and $16.4 \%$ of the total emissions respectively [14].

An emission inventory can show the temporal and spatial distributions and changes in pollutants in a certain area over a period of time. Currently, the global level non-road machinery activities and emission data are difficult to obtain, and the development of emission models and inventories is still being undertaken. Only the NONROAD model, developed by the U.S. Environmental Protection Agency (EPA), has been widely used [15]

Mobile sources can exhaust many kinds of pollutants including sulfur dioxide $\left(\mathrm{SO}_{2}\right)$, nitrogen oxides (NOx), total hydrocarbons (THC), carbon monoxide (CO) and particulate matter (PM)) during the process of fuel combustion. These pollutants can cause direct or indirect adverse influences to air pollution $[16,17,18]$, human health $[19,20]$ and climate change [21, 22, 23]. For example, emitted PM could directly increase atmospheric particulate matter with a diameter below $2.5 \mu \mathrm{m}$ (PM2.5) concentrations; as important fine particle precursors, $\mathrm{SO}_{2}$ and $\mathrm{NOx}$ can transform into sulfate and nitrate, making a secondary contribution to the PM2.5 [24, 25].

In this study, a diesel emission inventory for agricultural tractors including 2 wheeled driving (2WD) and 4 wheeled driving (4WD) tractors was established and analyzed using 2011 and 2019 data in Korea by categorizing tractors in terms of their rated powers. Also, emission characteristics of seven air pollutants from various field operations was analyzed. In addition, the spatial distribution of the amount of eight pollutants was visualized by geographic information system (GIS) and was investigated on country scale.

\section{Materials and Methods}

\subsection{Calculation of Air Pollutant Amount Emitted from Farm Tractor Operation}

Emitted amounts of seven air pollutant substances due to tractor operation were calculated by the method of NIER [26]. The formula is shown in Equation (1):

$$
E_{i, j, k}=\sum\left\{N_{i, k} \times H P_{i} \times L F \times H R S_{i} \times E F_{i, j}\right\}
$$

where, $E_{i, j, k}$ is Total amount of air pollutant emitted from specific region $(\mathrm{kg} / \mathrm{yr}) ; \mathrm{N}_{\mathrm{i}, \mathrm{k}}$ is Number of machinery of specific region (unit); $\mathrm{HP}_{\mathrm{i}}$ is Average rated power of tractor $(\mathrm{kw})$; LF is Load factor $(=0.48) ; \mathrm{HRS}_{\mathrm{i}}$ is Average annual activity of tractor $(\mathrm{hr} / \mathrm{yr}) ; \mathrm{EF}_{\mathrm{i}, \mathrm{j}}$ is Emission factor ( $\mathrm{kg} /(\mathrm{kWh}$-unit)); $\mathrm{i}$ is Farm tractor type (walking, riding)(i=1, $\cdots, 4) ; \mathrm{j}$ is type of air pollutant $(\mathrm{j}=1, \cdots, 8) ; \mathrm{k}$ is region $(\mathrm{k}=1, \cdots, 10)$.

The NIER handbook gives the emission factors for CO, NOx, TSP, PM2.5, VOCs, and $\mathrm{NH}_{3}$ but the following equation is used to calculate the emission factor for SOx [21].

$$
\mathrm{EF}_{\mathrm{i}}=\mathrm{FF}_{\mathrm{i}}(\mathrm{g} / \mathrm{kWh} \text {-unit }) / 1000 \times 1 \times \text { Fuel sulfur weight percent }(\%) / 100
$$

where, $\mathrm{EF}_{\mathrm{i}}$ is Emission factor $(\mathrm{kg} /(\mathrm{kWh}-\mathrm{unit})) ; \mathrm{FF}_{\mathrm{i}}$ is Fuel factor $(\mathrm{g} /(\mathrm{kWh}-\mathrm{unit})) ; 1$ is constant $(=2.0)$ (grams of SOx formed from one gram of sulfur); $i$ is Farm tractor type

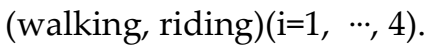

\subsection{Number of Farm Tractors}

In agricultural machinery statistic data from the Ministry of Agriculture, Food and Rural Affairs (MAFRA) in Korea, the number of agricultural tractors were categorized into two groups, a power tiller so called as a walking $2 \mathrm{WD}$ tractor and a farm tractor known as a riding $4 \mathrm{WD}$ tractor. The farm tractor group contains three subgroups, small, medium, large, categorized by tractor's engine rated powers. The Table 1 shows statistic 
data of tractors used in Korean agriculture in 2011 and 2019. Table 1 shows the number of tractors each year in Korea. Table 2 shows the average rated powers of diesel tractors used for calculating diesel emissions.

Table 1. Registration status of farm tractor in Korea.

\begin{tabular}{lccc}
\hline \multirow{2}{*}{ Tractor Type } & $\begin{array}{c}\text { Engine Size } \\
\text { (rated power range) }\end{array}$ & \multicolumn{2}{c}{ Unit (ea) } \\
\cline { 3 - 4 } Power tiller & - & 666,897 & $\mathbf{2 0 1 9}$ \\
& $\mathrm{S}^{1}(\mathrm{~S} \leq 29.4 \mathrm{~kW})$ & 73,901 & 73,604 \\
Farm Tractor & $\mathrm{M}^{2}(29.4 \mathrm{~kW} \leq \mathrm{M}<44.1 \mathrm{~kW})$ & 142,856 & 150,283 \\
& $\mathrm{~L}^{3}(44.1 \mathrm{~kW} \leq \mathrm{L})$ & 51,114 & 74,793 \\
& $\mathrm{Sub}$ Total & 267,871 & 298,680 \\
\hline
\end{tabular}

${ }^{1}$ S: Small, ${ }^{2}$ M: Medium, ${ }^{3}$ L: Large.

Table 2. Average rated power of tractors.

\begin{tabular}{ccc}
\hline Tractor Type & $\begin{array}{c}\text { Engine Size } \\
\text { (rated power range) }\end{array}$ & ARP $^{4} \mathbf{( k W )}$ \\
\hline Power tiller & - & 6.7 \\
\hline \multirow{2}{*}{ Farm Tractor } & $\mathrm{S}^{1}(\mathrm{~S}<29.4 \mathrm{~kW})$ & 23.0 \\
& $\mathrm{M}^{2}(29.4 \mathrm{~kW} \leq \mathrm{M}<44.1 \mathrm{~kW})$ & 39.0 \\
& $\mathrm{~L}^{3}(44.1 \mathrm{~kW} \leq \mathrm{L})$ & 52.1 \\
\hline
\end{tabular}

${ }^{1}$ S: Small, ${ }^{2}$ M: Medium, ${ }^{3}$ L: Large, ${ }^{4}$ ARP: Average rated power.

\subsection{Average Annual Operating Hours of Farm Tractor}

Average annual operating hours are given in Table 3 according to tractor types as well as some typical agricultural practices. Survey data by the Rural Development Administration (RDA) on the utilization of agricultural machinery were used to identify the average annual operating hours of agricultural tractors [4, 27].

Table 3. Average annual operating hours of farm tractors.

\begin{tabular}{|c|c|c|c|}
\hline \multirow{2}{*}{$\begin{array}{c}\text { Tractor } \\
\text { type }\end{array}$} & \multirow{2}{*}{ Operation Type } & \multicolumn{2}{|c|}{ Average Annual Activity (hr/yr) } \\
\hline & & 2011 & 2019 \\
\hline \multirow{7}{*}{$\begin{array}{c}\text { Power } \\
\text { Tiller }\end{array}$} & $\mathrm{TL}^{1}$ & 3.7 & 1.4 \\
\hline & $\mathrm{HW}^{2}$ & 6.1 & 3.8 \\
\hline & $\mathrm{PP}^{3}$ & 9.1 & 10.5 \\
\hline & $\mathrm{SY}^{4}$ & 25.2 & 19.9 \\
\hline & $\mathrm{TP}^{5}$ & 56.5 & 34.3 \\
\hline & Others & 0.2 & 2.3 \\
\hline & Total & 100.8 & 72.2 \\
\hline \multirow{10}{*}{$\begin{array}{l}\text { Farm } \\
\text { Tractor }\end{array}$} & TL $^{1}$ & 21.1 & 18.1 \\
\hline & $\mathrm{HW}^{2}$ & 31.5 & 44.6 \\
\hline & LL 6 & 11.7 & 14.1 \\
\hline & FS 7 & 6.9 & 8.2 \\
\hline & $\mathrm{CS}^{8}$ & 16.6 & 5.7 \\
\hline & $\operatorname{LD}^{9}$ & 13.2 & 23.4 \\
\hline & BL 10 & 6.9 & 2.7 \\
\hline & $\mathrm{TP} 5$ & 29.1 & 14.0 \\
\hline & Other & 13.9 & 9.1 \\
\hline & Total & 153.7 & 139.9 \\
\hline
\end{tabular}

${ }^{1}$ TL: Tilling, ${ }^{2}$ HW: Harrowing, ${ }^{3}$ PP: Pumping, ${ }^{4}$ SY: Spraying, ${ }^{5}$ TP: Transporting, 
${ }^{6}$ LL: Leveling, ${ }^{7}$ FS: Fertilizer spreading, ${ }^{8}$ CS: Compost spreading, ${ }^{9}$ LD: Loading, ${ }^{10}$ BL: Baling.

\subsection{Emission Factor of Farm Tractor}

Table 4 shows emission and fuel factors required to calculate the amount of six air pollutants emitted from agricultural tractors.

Table 4. Emission Factors of Six Air Pollutants of Agricultural Tractors.

\begin{tabular}{ccccccccc}
\hline \multirow{2}{*}{ Tractor Type } & Size & \multicolumn{6}{c}{ Emission Factor(kg/kWh-unit) } \\
\cline { 3 - 8 } & & CO & NOx & $\begin{array}{c}\text { TSP } \\
\left(\mathbf{P M}_{10}\right)\end{array}$ & $\mathbf{P M}_{2.5}$ & VOCs & $\mathbf{N H}_{3}$ & $\begin{array}{c}\text { Fuel } \\
\text { Factor }\end{array}$ \\
\hline Power Tiller & & 6.80 & 13.60 & 1.36 & 1.251 & 2.04 & 0.00004 & 0.00542 \\
\hline Farm & $\mathrm{S}^{1}$ & & & & & & & 0.00538 \\
Tractor & $\mathrm{M}^{2}$ & 2.48 & 7.84 & 0.39 & 0.359 & 0.48 & 0.00003 & 0.00530 \\
& $\mathrm{~L}^{3}$ & & & & & & & 0.00530 \\
\hline
\end{tabular}

${ }^{1}$ S: Small, ${ }^{2}$ M: Medium, ${ }^{3}$ L: Large. Total Suspended Particles (TSP) includes PM10.

\subsection{Visualization of Emissions}

To calculate the domestic spatial distribution of total air pollutant emissions from tractors, an open source geographic information system (GIS) software (QGIS, Windows 10 version) was used. The total of 9 provinces and 1 total metropolitan city (TMC) including 8 metropolitan cities was analyzed in the study. Based on the geocoded residential addresses, individual exposure to the various variables was assessed applying GIS program. QGIS is a widely-used, open-source GIS visualization tool that allows users to produce, edit, visualize and analyze spatial data. It supports vector and raster format, as well as a database format and functionalities (QGIS, 2021).

\section{Results and Discussion}

\subsection{Total Emissions of Agricultural Tractors}

The air pollutant emission inventory for agricultural tractors in 2011 and 2019 in Korea was calculated. Tables 5 and 6 show that the amount of air pollutants emitted from various agricultural practices from agricultural tractors. The two main sources of air pollutants emitted from walking tractor are transporting and spraying. They emit about $81 \%$ of emission from waling tractors. Riding tractors are heavily used in soil preparation, which emits about $42 \%$ of air pollutants, including tilling, harrowing and leveling, for seeding and planting in Korea. Figure 1 shows the change of calculated total emissions in agricultural tractors categorized into 4 groups. The total amount of emission using agricultural tractors was decreased about 13\% from 2011 to 2019. The number of power tillers were decreased by about $19 \%$ in 8 years, on the other hand that of farm tractors were increased by about $12 \%$. However, the emission reduction is about $48 \%$ for power tillers and the emission increment is about $5 \%$ for farm tractors. Thus, the total emission from agricultural tractors was decreased by about $16 \%$ in those periods. It is due to the decrease of $21 \%$ and $15 \%$ in the hours of use of walking and riding tractors, respectively, in 2019. However, the total amount of air pollutants emitted from large size riding tractors was increased by about $33 \%$ in 2019. Recently, Korean farmers are showing a tendency of using large size riding tractors to reduce working hours in the fields.

Table 5 . Calculated amounts of air pollutants emitted from agricultural tractors operations in 2011.

\begin{tabular}{ccccccccc}
\hline Machinery & $\begin{array}{c}\text { Operation } \\
\text { Type }\end{array}$ & CO & NOx & $\begin{array}{c}\text { SOx } \\
\left(\times \mathbf{1 0}^{2}\right)\end{array}$ & TSP & $\mathbf{P M}_{2.5}$ & VOCs & $\begin{array}{c}\mathbf{N H}_{3} \\
(\times \mathbf{1 0})\end{array}$ \\
\hline Walking Tractor & $\mathrm{TL}^{1}$ & 53.9 & 107.8 & 2.88 & 10.78 & 9.92 & 16.17 & 3.17
\end{tabular}




\begin{tabular}{|c|c|c|c|c|c|c|c|c|c|}
\hline \multicolumn{2}{|c|}{ (Power Tiller) } & $\mathrm{HW}^{2}$ & 88.9 & 177.7 & 4.75 & 17.77 & 16.35 & 26.7 & 523 \\
\hline & & $\mathrm{PP}^{3}$ & 132.6 & 265 & 7.08 & 26.5 & 24.4 & 39.8 & 7.80 \\
\hline & & SY 4 & 367 & 734 & 19.61 & 73.4 & 67.5 & 110.1 & 21.6 \\
\hline & & $\mathrm{TP}^{5}$ & 823 & $1,646.3$ & 44.0 & 164.6 & 151.4 & 246.9 & 48.4 \\
\hline & & Other & 2.91 & 5.83 & 0.16 & 0.583 & 0.536 & 0.874 & 0.0171 \\
\hline & & SubTotal & 1,469 & 2,940 & 78.4 & 296 & 270 & 441 & 86.2 \\
\hline \multirow{32}{*}{$\begin{array}{l}\text { Riding } \\
\text { Tractor }\end{array}$} & \multirow{10}{*}{ Small } & $\mathrm{TL}^{1}$ & 427 & 135.1 & 6.21 & 6.7 & 6.2 & 8.3 & 5.17 \\
\hline & & $\mathrm{HW}^{2}$ & 63.9 & 202 & 9.28 & 10.0 & 9.2 & 124 & 7.73 \\
\hline & & $\mathrm{LL}^{6}$ & 23.7 & 74.9 & 3.44 & 3.7 & 3.4 & 4.6 & 287 \\
\hline & & FS 7 & 14.0 & 44.2 & 2.03 & 2.2 & 20 & 2.7 & 1.690 \\
\hline & & $\mathrm{CS}^{8}$ & 33.6 & 1063 & 4.89 & 5.3 & 49 & 6.5 & 4.07 \\
\hline & & $\mathrm{LD}^{9}$ & 26.7 & 84.5 & 3.89 & 4.2 & 3.9 & 52 & 3.23 \\
\hline & & BL 10 & 19.6 & 621 & 2.86 & 3.1 & 28 & 3.8 & 2.38 \\
\hline & & $\mathrm{TP}^{5}$ & 58.9 & 186.3 & 8.57 & 9.3 & 8.5 & 11.4 & 7.13 \\
\hline & & Other & 28.2 & 89.0 & 4.09 & 4.43 & 4.07 & 5.45 & 3.41 \\
\hline & & SubTotal & 311 & 984 & 45.3 & 49.0 & 45.1 & 60.3 & 37.7 \\
\hline & \multirow{10}{*}{ Medium } & $\mathrm{TL}^{1}$ & 140.0 & 442 & 20.0 & 220 & 20.2 & 27.1 & 16.90 \\
\hline & & $\mathrm{HW}^{2}$ & 209 & 661 & 29.9 & 329 & 30.3 & 40.5 & 25.3 \\
\hline & & LL $^{6}$ & 77.6 & 245 & 11.1 & 122 & 11.20 & 15.00 & 9.38 \\
\hline & & FS 7 & 45.7 & 145.0 & 6.55 & 7.19 & 6.62 & 8.85 & 5.53 \\
\hline & & $\mathrm{CS}^{8}$ & 110.0 & 348 & 15.80 & 17.30 & 15.90 & 21.3 & 13.30 \\
\hline & & $\mathrm{LD}^{9}$ & $87.5 \pm$ & 277 & 12.50 & 13.80 & 1270 & 16.9 & 10.60 \\
\hline & & BL 10 & 643 & 203 & 921 & 10.10 & 9.31 & 124 & 7.78 \\
\hline & & $\mathrm{TP}^{5}$ & 193.0 & 610 & 27.6 & 30.30 & 27.9 & 37.3 & 23.3 \\
\hline & & Other & 921 & 291 & 13.20 & 14.50 & 13.30 & 17.8 & 11.10 \\
\hline & & SubTotal & 1,019 & 3,220 & 145.9 & 160.3 & 1475 & 197.3 & 123.3 \\
\hline & \multirow{10}{*}{ Large } & $\mathrm{TL}^{1}$ & 66.9 & 211 & 9.57 & 10.50 & 9.68 & 1290 & 8.09 \\
\hline & & $\mathrm{HW}^{2}$ & 99.9 & 316 & 14.30 & 15.70 & 14.50 & 19.30 & 1210 \\
\hline & & $\mathrm{LL}^{6}$ & 37.1 & 117.0 & 5.31 & 5.83 & 5.37 & 7.18 & 4.48 \\
\hline & & FS ${ }^{7}$ & 21.9 & 69.1 & 3.13 & 3.44 & 3.16 & 4.23 & 2.64 \\
\hline & & $\mathrm{CS}^{8}$ & 526 & 166.0 & 7.53 & 8.27 & 7.61 & 10.20 & 6.36 \\
\hline & & $\mathrm{LD}^{9}$ & 41.8 & 1320 & 5.99 & 6.58 & 6.05 & 8.90 & 5.06 \\
\hline & & BL 10 & 30.7 & 97.2 & 4.40 & 4.83 & 4.45 & 5.95 & 3.72 \\
\hline & & $\mathrm{TP}^{5}$ & 922 & 291 & 13.20 & 14.50 & 13.30 & 17.80 & 11.20 \\
\hline & & Other & 44.0 & 139.0 & 6.31 & 6.93 & 6.38 & 8.52 & 5.33 \\
\hline & & SubTotal & 487 & 1,540 & 69.7 & 76.6 & 70.5 & 94.3 & 58.9 \\
\hline & \multicolumn{2}{|c|}{ Sub Total } & 1,818 & 5,746 & 261 & 286 & 267 & 352 & 220 \\
\hline & \multicolumn{2}{|l|}{ Total } & 3,290 & 8,683 & 339 & 580 & 537 & 792 & 306 \\
\hline
\end{tabular}

${ }_{1}^{1}$ TL: Tilling, ${ }^{2}$ HW: Harrowing, ${ }^{3}$ PP: Pumping, ${ }^{4}$ SY: Spraying, ${ }^{5}$ TP: Transporting, ${ }^{6}$ LL: Leveling, ${ }^{7}$ FS: Fertilizer spreading, ${ }^{8}$ CS: Compost spreading, ${ }^{9}$ LD: Loading, ${ }^{10}$ BL: Baling.

Table 6 . Calculated amounts of air pollutants emitted from agricultural tractors operations in 2019.

\begin{tabular}{cccccccccc}
\hline & \multicolumn{1}{c}{ Machinery } & $\begin{array}{c}\text { Operation } \\
\text { Type }\end{array}$ & CO & NOx & $\begin{array}{c}\text { SOx } \\
\left(\times \mathbf{1 0}^{2}\right)\end{array}$ & TSP & $\mathbf{P M}_{\mathbf{2 . 5}}$ & VOCs & $\begin{array}{c}\mathbf{N H}_{\mathbf{3}} \\
(\times \mathbf{1 0})\end{array}$ \\
\hline Walking Tractor & $\mathrm{TL}^{1}$ & 16.64 & 33.3 & 0.900 & 3.33 & 3.06 & 4.99 & 0.980 \\
(Power Tiller) & $\mathrm{HW}^{2}$ & 40.4 & 80.8 & 2.20 & 8.08 & 7.43 & 1212 & 2.38 \\
& $\mathrm{PP}^{3}$ & 124.8 & 250 & 6.70 & 25.0 & 23.0 & 37.4 & 7.34
\end{tabular}




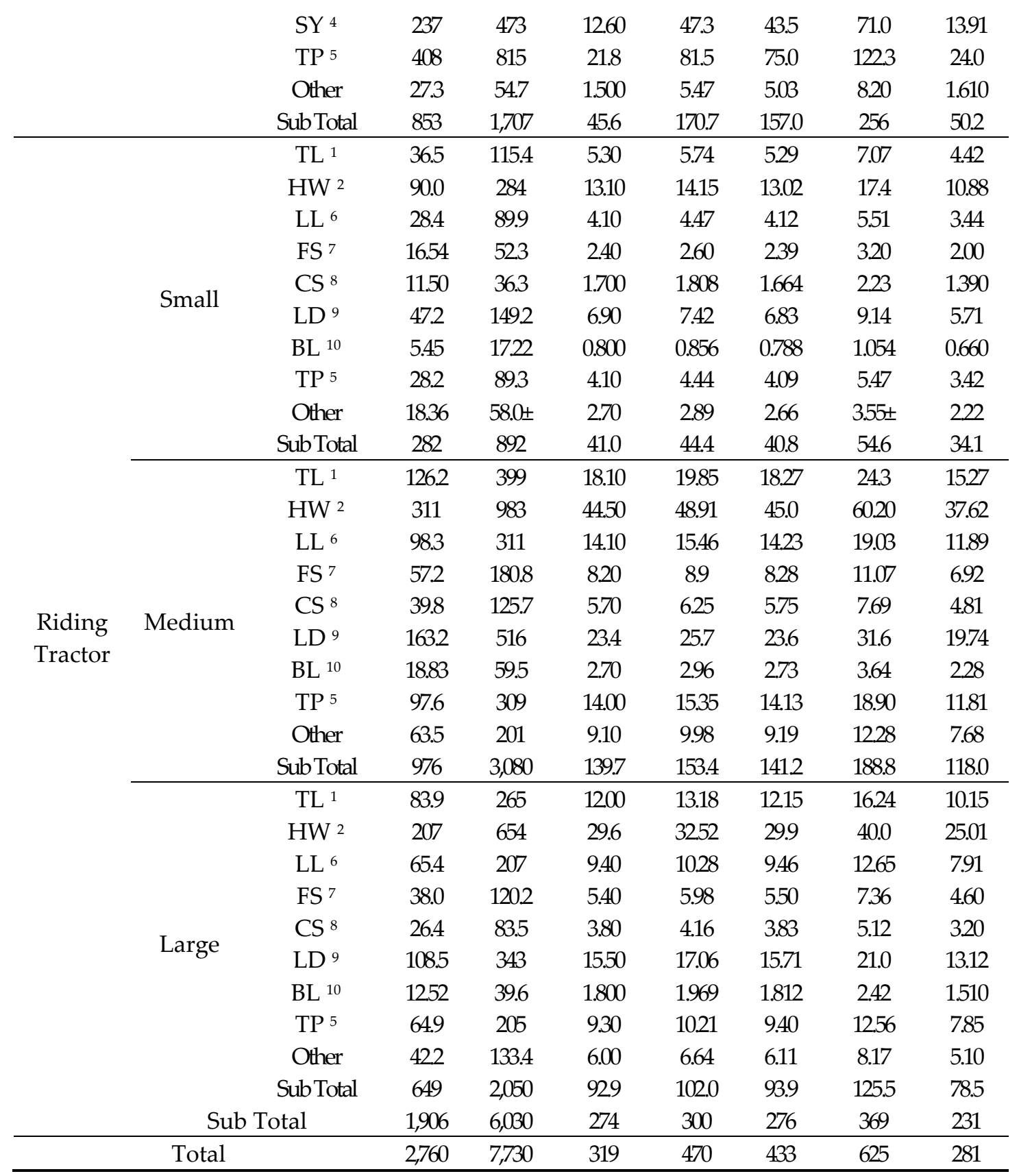

${ }_{1}^{1}$ TL: Tilling, ${ }^{2}$ HW: Harrowing, ${ }^{3}$ PP: Pumping, ${ }^{4}$ SY: Spraying, ${ }^{5}$ TP: Transporting, ${ }^{6}$ LL: Leveling, ${ }^{7}$ FS: Fertilizer spreading, 8 CS: Compost spreading, ${ }^{9}$ LD: Loading, ${ }^{10}$ BL: Baling. 


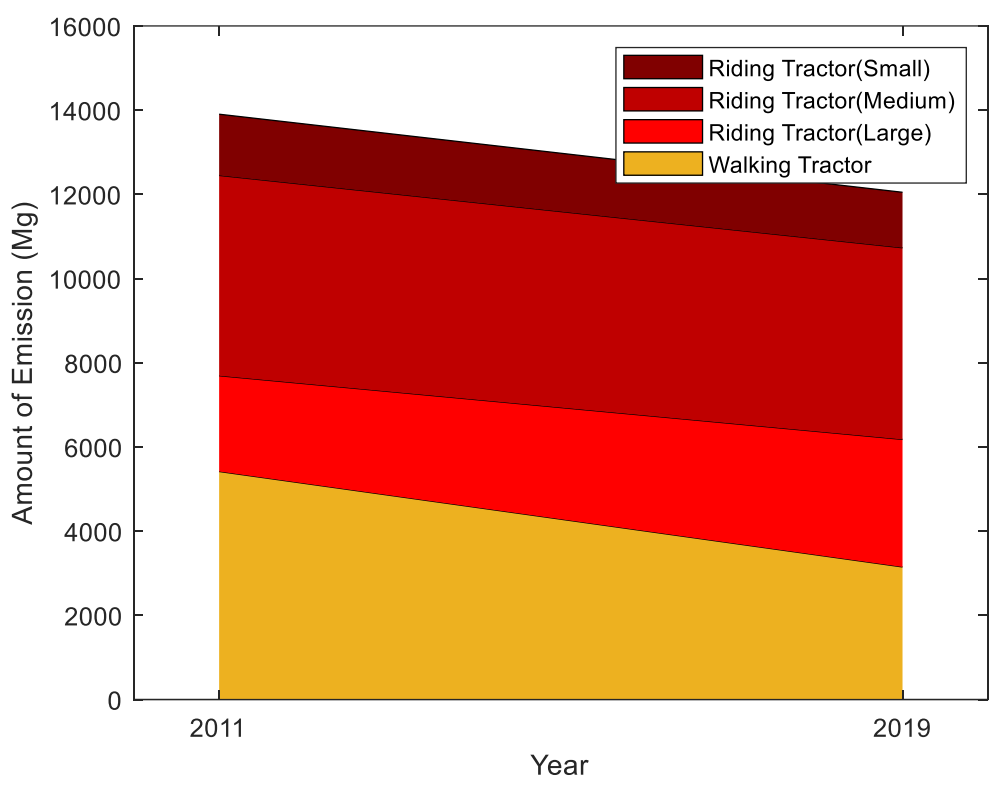

Figure 1. Calculated average total air pollutant substances emitted from walking and riding tractors.

\subsection{Air Pollution Emissions from Farm Tractor Operations}

The diesel emission inventory for agricultural tractors in Korea was refined by categorizing the rated power of riding tractors into 3 sub-groups. Figures 2 and 3 show that calculated amounts of average air pollutants emission from various agricultural operations by walking and riding tractors, respectively. Walking tractors mainly emit air pollutants from spraying and transporting. In particular, in the case of transporting, 2,639 Mg of air pollutants was emitted in 2011 and 1,429 Mg of air pollutants was emitted in 2019. Riding tractors mainly emit air pollutants from soil preparation and transporting operations. About $61 \%$ of total air pollutants are emitted from those operations. Particularly air pollutant emission from loading in 2019 was nearly doubled compared to 2011. Korean farmers are using riding tractors as loading heavy material recently.
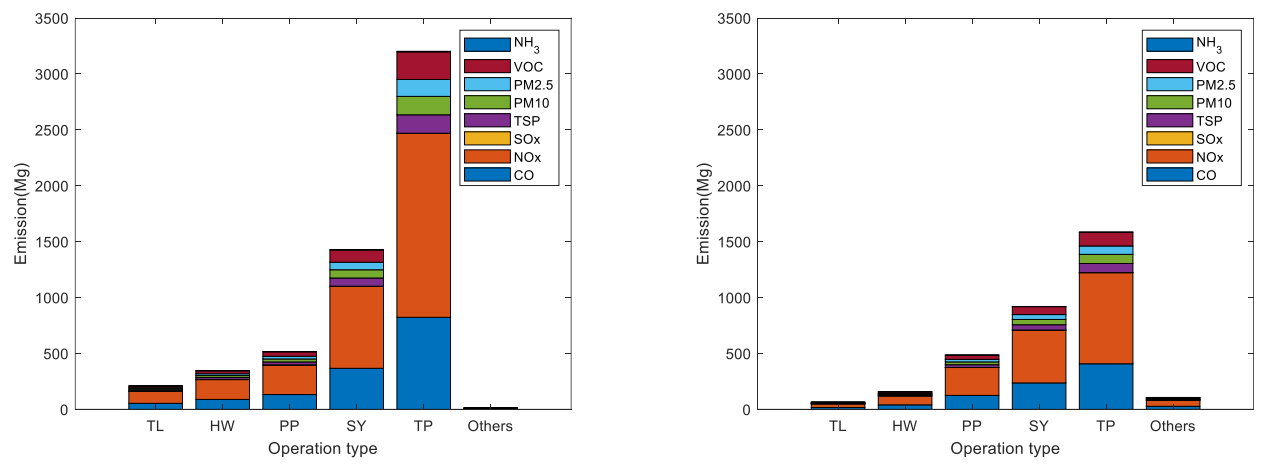

Figure 2. Calculated amounts of average air pollutant substances emission of from various agricultural operations by walking tractor in 2011 (left) and 2019 (right). TL: Tilling, HW: Harrowing, PP: Pumping, SY: Spraying, TP: Transporting 

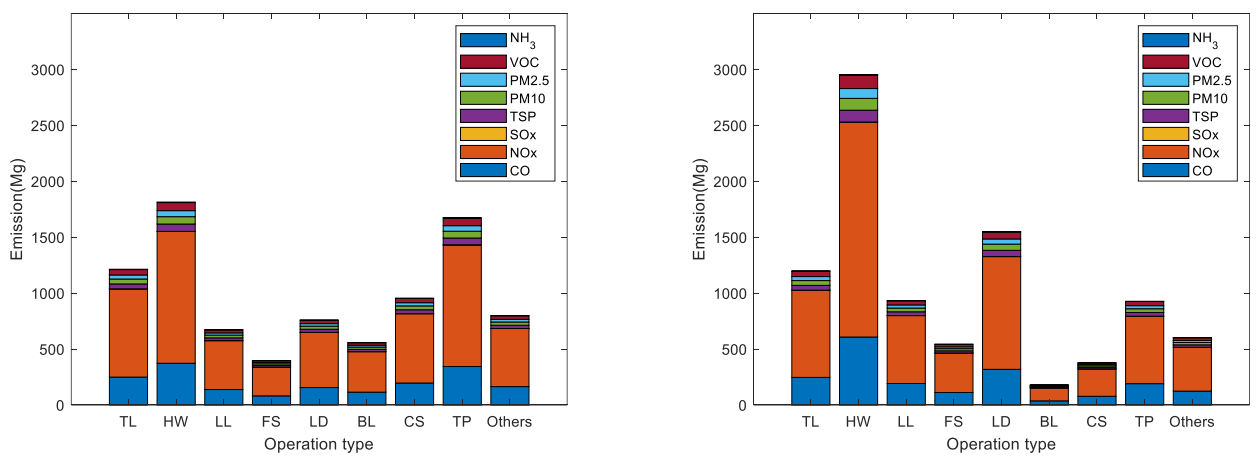

Figure 3. Calculated amounts of average air pollutant substances emission of from various agricultural operations by riding tractor in 2011 (left) and 2019 (right). TL: Tilling, HW: Harrowing, LL: Leveling, FS: Fertilizer spreading, LD: Loading, BL: Baling, CS: Compost spreading, TP: Transporting

\subsection{Spatial and Temporal Distribution of Air Pollutant Emissions}

The amount of air pollutants emitted by Korean agricultural tractors was calculated on a region-level. Tables 7, 8, 9, and 10 show the calculated air pollutant emissions of 10 regions in Korea. Total emissions of $\mathrm{CO}, \mathrm{NOx}, \mathrm{SOx}, \mathrm{TSP}, \mathrm{PM}_{2.5}$, VOCs and $\mathrm{NH}_{3}$ from walking tractors in 2011 were $1,469 \mathrm{Mg}, 2,940 \mathrm{Mg}, 0.78 \mathrm{Mg}, 296 \mathrm{Mg}, 270 \mathrm{Mg}$, $441 \mathrm{Mg}$ and $8.6 \mathrm{Mg}$, respectively. Also, total emissions of CO, NOx, SOx, TSP, PM2.5, VOCs and $\mathrm{NH}_{3}$ from walking tractors in 2019 were $853 \mathrm{Mg}, 1,716 \mathrm{Mg}$, $0.46 \mathrm{Mg}, 171 \mathrm{Mg}, 157 \mathrm{Mg}, 257 \mathrm{Mg}$ and $5.0 \mathrm{Mg}$, respectively. In 2019, walking tractor emissions of Gyeongsangbuk-do, Jeollanam-do, and Gyeongsangnam-do account for $23 \%, 16 \%$ and $13 \%$ of the total emissions. Total emissions of $\mathrm{CO}, \mathrm{NOx}, \mathrm{SOx}, \mathrm{TSP}, \mathrm{PM}_{2.5}, \mathrm{VOCs}$ and $\mathrm{NH}_{3}$ from riding tractors were $1,818 \mathrm{Mg}, 5,746 \mathrm{Mg}, 3 \mathrm{Mg}, 286 \mathrm{Mg}, 263 \mathrm{Mg}, 352 \mathrm{Mg}$ and $22 \mathrm{Mg}$, respectively in 2011 and were $1,906 \mathrm{Mg}, 6,030 \mathrm{Mg}, 3 \mathrm{Mg}, 300 \mathrm{Mg}, 276 \mathrm{Mg}$, $369 \mathrm{Mg}$ and $23 \mathrm{Mg}$, respectively in 2019. Gyeongsangbukdo and Jeollanam-do are major regions emitting about $30 \%$ and $32 \%$ of total air pollutants utilizing walking and riding tractors in 2019.

Table 7. Calculated air pollutant emissions of walking tractor by region in Korea (2011).

\begin{tabular}{|c|c|c|c|c|c|c|c|c|}
\hline \multirow{2}{*}{ Region } & \multicolumn{8}{|c|}{ Emission $(\mathrm{Mg} / \mathrm{yr})$} \\
\hline & $\mathrm{CO}$ & NOx & $\operatorname{SOx}\left(\times 10^{2}\right)$ & TSP & $\mathbf{P M}_{2.5}$ & VOCs & $\mathrm{NH}_{3}(\times 10)$ & Total \\
\hline $\mathrm{CHB}^{1}$ & 100 & 199 & 5 & 20 & 18 & 30 & 6 & 349 \\
\hline $\mathrm{CHN}^{2}$ & 192 & 384 & 10 & 38 & 35 & 58 & 11 & 673 \\
\hline GAW $^{3}$ & 85 & 171 & 5 & 17 & 16 & 26 & 5 & 299 \\
\hline GYB $^{4}$ & 306 & 613 & 16 & 61 & 56 & 92 & 18 & 1,074 \\
\hline GYG $^{5}$ & 129 & 258 & 7 & 26 & 24 & 39 & 8 & 453 \\
\hline GYN 6 & 195 & 390 & 10 & 39 & 36 & 58 & 11 & 684 \\
\hline JEB 7 & 133 & 265 & 7 & 27 & 24 & 40 & 8 & 465 \\
\hline JEJ 8 & 32 & 65 & 2 & 6 & 6 & 10 & 2 & 114 \\
\hline $\mathrm{JEN}^{9}$ & 232 & 464 & 12 & 46 & 43 & 70 & 14 & 814 \\
\hline TMC 10 & 64 & 128 & 3 & 13 & 12 & 19 & 4 & 225 \\
\hline Total & 1,469 & 2,940 & 78 & 296 & 270 & 441 & 86 & 5,425 \\
\hline
\end{tabular}

${ }^{1} \mathrm{CHB}$ : Chungcheongbuk-do, ${ }^{2} \mathrm{CHN}$ : Chungcheongnam-do, ${ }^{3} \mathrm{GAW}$ : Gangwon-do,

${ }^{4}$ GYB: Gyeong sangbuk-do, ${ }^{5}$ GYG: Gyeonggi-do, ${ }^{6}$ GYN: Gyeongsangnam-do,

${ }^{7}$ JEB: Jeollabuk-do, ${ }^{8}$ JEJ: Jeju-do, ${ }^{9}$ JEN: Jeollanam-do, ${ }^{10}$ TMC: Total of 8 metropolitan city

Table 8. Calculated air pollutant emissions of walking tractor by region in Korea (2019).

\begin{tabular}{ccccccccc}
\hline \multirow{2}{*}{ Region } & \multicolumn{7}{c}{ Emission $(\mathbf{M g} / \mathbf{y r})$} \\
\cline { 2 - 8 } & $\mathbf{C O}$ & NOx & $\mathbf{S O x}\left(\times \mathbf{1 0}^{\mathbf{2}}\right)$ & TSP & PM $_{2.5}$ & VOCs & $\mathbf{N H}_{3}(\times \mathbf{1 0})$ & Total \\
\hline $\mathrm{CHB}^{1}$ & 58 & 116 & 3 & 12 & 11 & 18 & 3 & 205 \\
$\mathrm{CHN}^{2}$ & 100 & 199 & 5 & 20 & 18 & 30 & 6 & 351 \\
\hline
\end{tabular}




\begin{tabular}{ccccccccc}
\hline GAW $^{3}$ & 50 & 100 & 3 & 10 & 9 & 15 & 3 & 177 \\
GYB $^{4}$ & 195 & 389 & 10 & 39 & 36 & 57 & 11 & 683 \\
GYG $^{5}$ & 64 & 127 & 3 & 13 & 12 & 19 & 4 & 224 \\
GYN $^{6}$ & 113 & 225 & 6 & 23 & 21 & 34 & 7 & 397 \\
JEB $^{7}$ & 76 & 151 & 4 & 15 & 14 & 23 & 4 & 267 \\
JEJ $^{8}$ & 23 & 46 & 1 & 5 & 4 & 7 & 1 & 80 \\
JEN $^{9}$ & 135 & 270 & 7 & 27 & 25 & 41 & 8 & 475 \\
TMC $^{10}$ & 43 & 84 & 2 & 25 & 8 & 13 & 3 & 151 \\
Total & 853 & 1,707 & 46 & 171 & 157 & 256 & 50 & 3,149 \\
\hline
\end{tabular}

${ }^{1}$ CHB: Chungcheongbuk-do, ${ }^{2} \mathrm{CHN}$ : Chungcheongnam-do, ${ }^{3} \mathrm{GAW}$ : Gangwon-do,

${ }^{4}$ GYB: Gyeong sangbuk-do, ${ }^{5}$ GYG: Gyeonggi-do, ${ }^{6}$ GYN: Gyeongsangnam-do,

${ }^{7}$ JEB: Jeollabuk-do, ${ }^{8}$ JEJ: Jeju-do, ${ }^{9}$ JEN: Jeollanam-do, ${ }^{10}$ TMC: Total of 8 metropolitan city

Table 9. Calculated air pollutant emissions of riding tractor by region in Korea (2011).

\begin{tabular}{|c|c|c|c|c|c|c|c|c|}
\hline \multirow{2}{*}{ Region } & \multicolumn{8}{|c|}{ Emission (Mg/yr) } \\
\hline & $\mathrm{CO}$ & NOx & $\operatorname{SOx}\left(\times 10^{2}\right)$ & TSP & $\mathbf{P M}_{2.5}$ & VOCs & $\mathrm{NH}_{3}(\times 10)$ & Total \\
\hline $\mathrm{CHB}^{1}$ & 108 & 342 & 16 & 17 & 16 & 21 & 13 & 490 \\
\hline $\mathrm{CHN}^{2}$ & 253 & 801 & 36 & 40 & 38 & 49 & 31 & 1,148 \\
\hline GAW 3 & 127 & 402 & 18 & 20 & 18 & 25 & 15 & 576 \\
\hline GYB 4 & 270 & 854 & 39 & 43 & 39 & 52 & 33 & 1,223 \\
\hline $\mathrm{GYG}^{5}$ & 259 & 819 & 37 & 41 & 38 & 50 & 31 & 1,173 \\
\hline GYN 6 & 207 & 655 & 30 & 33 & 31 & 40 & 25 & 939 \\
\hline $\mathrm{JEB}^{7}$ & 210 & 664 & 30 & 33 & 30 & 41 & 25 & 951 \\
\hline JEJ 8 & 30 & 94 & 4 & 5 & 4 & 6 & 4 & 134 \\
\hline JEN 9 & 273 & 864 & 39 & 43 & 41 & 53 & 33 & 1,238 \\
\hline TMC 10 & 79 & 249 & 11 & 12 & 11 & 15 & 10 & 357 \\
\hline Total & 1,818 & 5,746 & 261 & 286 & 267 & 352 & 220 & 8,493 \\
\hline
\end{tabular}

${ }^{1} \mathrm{CHB}$ : Chungcheongbuk-do, ${ }^{2} \mathrm{CHN}$ : Chungcheongnam-do, ${ }^{3} \mathrm{GAW}$ : Gangwon-do,

${ }^{4}$ GYB: Gyeong sangbuk-do, ${ }^{5}$ GYG: Gyeonggi-do, ${ }^{6}$ GYN: Gyeongsangnam-do,

${ }^{7}$ JEB: Jeollabuk-do, ${ }^{8}$ JEJ: Jeju-do, ${ }^{9}$ JEN: Jeollanam-do, ${ }^{10}$ TMC: Total of 8 metropolitan city

Table 10. Calculated air pollutant emissions of riding tractor by region in Korea (2019).

\begin{tabular}{|c|c|c|c|c|c|c|c|c|}
\hline \multirow{2}{*}{ Region } & \multicolumn{8}{|c|}{ Emission (Mg/yr) } \\
\hline & $\mathrm{CO}$ & NOx & $\operatorname{SOx}\left(\times 10^{2}\right)$ & TSP & $\mathbf{P M}_{2.5}$ & VOCs & $\mathrm{NH}_{3}(\times 10)$ & Total \\
\hline $\mathrm{CHB}^{1}$ & 113 & 358 & 16 & 18 & 16 & 22 & 14 & 512 \\
\hline $\mathrm{CHN}^{2}$ & 256 & 810 & 37 & 40 & 37 & 50 & 31 & 1,160 \\
\hline GAW 3 & 137 & 433 & 20 & 22 & 20 & 27 & 17 & 620 \\
\hline $\mathrm{GYB}^{4}$ & 312 & 986 & 45 & 49 & 45 & 60 & 38 & 1,411 \\
\hline GYG $^{5}$ & 230 & 727 & 33 & 36 & 33 & 45 & 28 & 1,041 \\
\hline GYN 6 & 219 & 694 & 32 & 35 & 32 & 42 & 27 & 993 \\
\hline $\mathrm{JEB}^{7}$ & 228 & 720 & 33 & 36 & 33 & 44 & 28 & 1,030 \\
\hline $\mathrm{JEJ}^{8}$ & 32 & 103 & 5 & 5 & 5 & 6 & 4 & 147 \\
\hline JEN 9 & 291 & 919 & 42 & 46 & 42 & 56 & 35 & 1,315 \\
\hline TMC 10 & 88 & 278 & 13 & 14 & 13 & 17 & 11 & 398 \\
\hline Total & 1,906 & 6,030 & 274 & 300 & 276 & 369 & 231 & 8,906 \\
\hline
\end{tabular}

${ }^{1}$ CHB: Chungcheongbuk-do, ${ }^{2} \mathrm{CHN}$ : Chungcheongnam-do, ${ }^{3} \mathrm{GAW}$ : Gangwon-do,

${ }^{4}$ GYB: Gyeong sangbuk-do, ${ }^{5}$ GYG: Gyeonggi-do, ${ }^{6}$ GYN: Gyeongsangnam-do,

${ }^{7}$ JEB: Jeollabuk-do, ${ }^{8}$ JEJ: Jeju-do, ${ }^{9}$ JEN: Jeollanam-do, ${ }^{10}$ TMC: Total of 8 metropolitan city

The spatial distribution of the total amount of tractor air pollutant emission in Korea were visualized at the region-level using a GIS technique, as shown in Figures 4 and 5. Figure 4 (a) shows the emissions in 2011 and Figure 4 (b) shows the emissions in 2019 from walking tractors. Regions 
with high emissions are mainly southern regions such as: Gyeongsangbuk-do, Jeollanam-do, and Gyeongsangnam-do. In the case of Gyeongsangbuk-do compared to other regions, it emits about $22.7 \%$ of total air pollutants in 2019. Figure 5 (a), (b) shows the emissions from riding tractors in 2011 and 2019. Gyeongsangbuk-do and Jeollanam-do emit about 31.6\% of total air pollutants in 2019. Figures 4 and 5 show that the emission of the walking tractor is decreasing, but the emission of the riding tractor is increasing in Gyeongsangbuk-do region. In 2011, riding tractor emissions were in about same range in Chungcheongnam-do, Gyeongsangbuk-do, Gyeonggi-do, and Jeollanam-do, but in 2019, the emission range in Gyeongsangbuk-do, Jeollanam-do, Chungcheongbuk-do and Jeollabuk-do increased by one level. These high-emission areas are mainly located in the western and southern regions of Korea, which have plenty of arable areas compared to other regions in Korea. However, riding tractor emissions are much lower in Gangwon-do and Chungcheongbuk-do. This area is mainly mountainous, so there is little agricultural area to produce agricultural products. Metropolitan cities with high population generate less emissions from agricultural tractors compared to other regions.

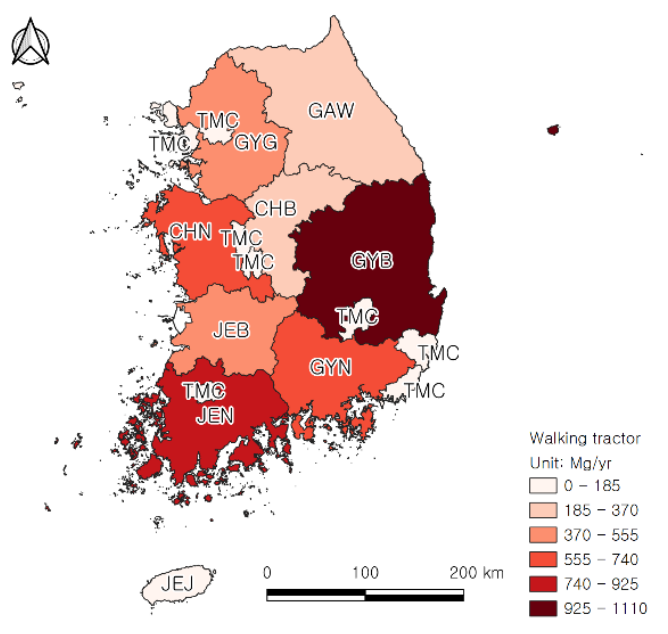

(a)



(b)

Figure 4. Spatial distribution of emissions from walking tractors in 2011 (left) and 2019 (right).

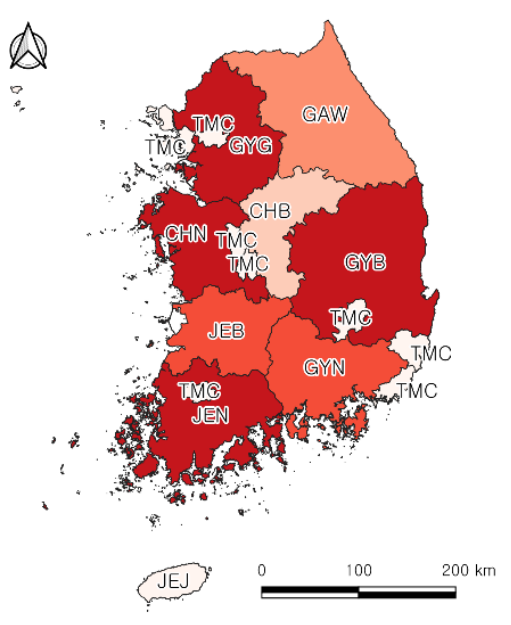

(a)
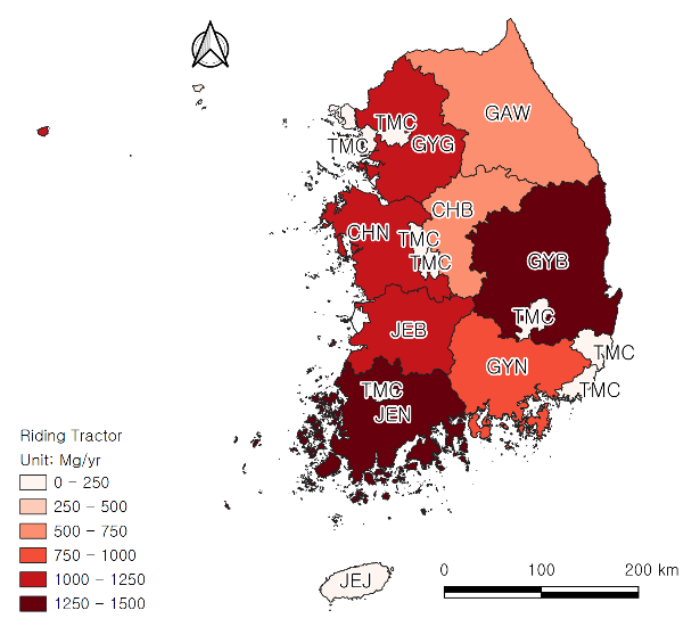

(b)

Figure 5. Spatial distribution of emissions from riding tractors in 2011 (left) and 2019 (right).

\section{Conclusions}

In this study, agricultural tractors including walking and riding types were categorized into 4 levels by rated output power and diesel emissions of tractors were analyzed 
using 2011 and 2019 survey data in Korea. Emission inventory including CO, NOx, SOx, TSP(PM10), $\mathrm{PM}_{2.5}$, VOCs and $\mathrm{NH}_{3}$ were established using Tier 3 methodology. As a result, the annual emissions of $\mathrm{CO}, \mathrm{NOx}, \mathrm{SOx}, \mathrm{TSP}\left(\mathrm{PM}_{10}\right), \mathrm{PM}_{2.5}$, VOCs and $\mathrm{NH}_{3}$ of agricultural tractors were $3,290 \mathrm{Mg}, 8,683 \mathrm{Mg}$, $3.39 \mathrm{Mg}, 580 \mathrm{Mg}$, $537 \mathrm{Mg}$, $792 \mathrm{Mg}$ and $30.6 \mathrm{Mg}$, respectively in 2011 and 2,760 Mg, 7,730 Mg, $3.19 \mathrm{Mg}$, $470 \mathrm{Mg}, 433 \mathrm{Mg}, 625 \mathrm{Mg}$ and $28.1 \mathrm{Mg}$, respectively in 2019. The total amount of emission using agricultural tractors was decreased about 13\% from 2011 to 2019. The number of walking tractors were decreased by about 19\% in 8 years, on the other hand that of riding tractors were increased by about $12 \%$. However, the emission reduction is about $48 \%$ for walking tractors and the emission increment is about $5 \%$ for riding tractors. Thus, the total emission from agricultural tractors was decreased by about $16 \%$ in those periods. It is due to the decrease of $21 \%$ and $15 \%$ in the hours of use of walking and riding tractors, respectively, in 2019. Walking tractors mainly emit air pollutants from spraying and transporting. In particular, in the case of transporting, 2,639 Mg of air pollutants was emitted in 2011 whereas 1,429 Mg of air pollutants was emitted in 2019. Riding tractors mainly emit air pollutants from soil preparation and transporting operations. About $61 \%$ of total air pollutants are emitted from those operations. High emission generating regions and changes of emissions during 8 years were clearly seen in GIS analysis. High air pollutant emitting regions are mainly located in the western and southern regions of Korea, which have plenty of arable areas compared to other regions in Korea.

Author Contributions: Conceptualization, G.G.H. and S.M.K.; software, G.G.H.; validation, G.G.H., M.H.K. and S.M.K.; investigation, J.H.J.; resources, J.M.L. and G.G.H.; writing-original draft preparation, G.G.H.; writing - review and editing, M.H.K. and S.M.K.; visualization, J.H.J.; supervision, S.M.K.; project administration, S.M.K.; funding acquisition, S.M.K. All authors have read and agreed to the published version of the manuscript.

Funding: This work was carried out with the support of "Study on Particulate Matter Outbreak Source Characteristics during Agricultural Practice and Inventory Integration (Project No. PJ01428301)" Rural Development Administration, Korea.

Institutional Review Board Statement: Not applicable.

Informed Consent Statement: Not applicable.

Conflicts of Interest: The funders had no role in the design of the study; in the collection, analyses, or interpretation of data; in the writing of the manuscript, or in the decision to publish the results.

\section{References}

1. MAFRA. 2019 Agricultural Area Survey, Ministry of Agriculture, Food and Rural Affairs, Sejong, Korea, 2020

2. MAFRA. 2019 Crop Production Survey, Ministry of Agriculture, Food and Rural Affairs, Sejong, Korea, 2020.

3. MAFRA. 2019 Agricultural Machinery Holdings Survey, Ministry of Agriculture, Food and Rural Affairs, Sejong, Korea, 2020.

4. RDA. 2019 Survey on the Utilization of Agricultural Machinery and Farmwork Mechanization Rate, Rural Development Administration, Jeonju, Korea, 2020.

5. Kim, J.G.; Park, J.S.; Choi, K.J.; Lee, D.K.; Shin, M.S.; Oh, J.Y.; Nam, J.S. Analysis of Agricultural Tractor Transmission using Actual Farm Workload. Journal of the Korean Society of Manufacturing Process Engineers. 2020, 19(11), 42-48.

6. Lee, N.G.; Kim, Y.J.; Beak, S.M.; Moon, S.P.; Park, S.U.; Choi, Y.S.; Cho C.H. Analysis of Traction of Agricultural Tractor According to Soil Condition. Journal of Drive and Control. 2020, 17(4), 133-140.

7. Lewis, P.; Frey, H.C.; Rasdorf, W. Development and use of emissions inventories for construction vehicles. Transp Res Rec 2009. 46-53.

8. Millstein, D.E.; Harley, R.A. Revised estimates of construction activity and emissions: effects on ozone and elemental carbon concentrations in southern California. Atmos Environ. 2009. 43,6328-635.

9. Lelicveld, J.; Evans, J.; Fnais, M.; Giannadaki, D.; Pozzer, A. The contribution of outdoor air pollution sources to premature mortality on a global scale. Nature. 2015. 525, 367-371.

10. Douglas, P.; Robertson, S.; Gay, R.; Hansell, A.L. Gant, T.W. A systematic review of the public health risks of bioaerosols from intensive farming. Int. J. Hyg Environ. Health. 2018. 221, 134-173. https://doi.org/10.1016/j.ijheh.2017.10.019.

11. Giannadaki, D.; Giannakis, E.; Pozzer, A.; Lelieveld, J. Estimating health and economic benefits of reductions in air pollution from agriculture. Sci. Total Environ. 2018. 622-623, 1304-1316. https://doi.org/10.1016/j.scitotenv.2017.12.064.

12. Giannakis, E., Kushta, J., Giannadaki, D., Georgiou, G.K., Bruggeman, A., Lelieveld, J. Exploring the economy-wide effects of agriculture on air quality and health: evidence from Europe. Sci. Total Environ. 2019, 663, 889-900. https://doi.org/10.1016/j. scitotenv.2019.01.410. 
13. Yang, W.H. Changes in Air Pollutant Concentrations Due to Climate Change and the Health Effect of Exposure to Particulate Matter. Korean Studies Information Service System. 2019. 269, 20-31.

14. NIER. 2017 National Air Pollutants Emission, National Institute of Environmental Research, Sejong, Korea, 2020. pp. 19.

15. Xiuning Hou, Jinling Tian, Changbo Song, Jie Wang, Jiyun Zhao, Xuemin Zhang. Emission inventory research of typical agricultural machinery in Beijing, China, 2019, 216.

16. Kim, H.H.; Byun, G.R.; Choi, Y.S.; Kim, S.R.; Kim S.Y.; Lee, J.T. Effects of long-term exposure to air pollution on all-cause mortality and cause-specific mortality in seven major cities of South Korea: Korean national health and nutritional examination surveys with mortality follow-up. Environmental Research. 2021. 192, 110290. https://doi.org/10.1016/j.envres.2020.110290.

17. Yu, G.E.; Park, S.S. Chemical characterization and source apportionment of PM2.5 at an urban site in Gwangju, Korea. Atmospheric Pollution Research. 2021. 12, 101092. https://doi.org/10.1016/j.apr.2021.101092.

18. Choi, S.W.; Kim, T.K.; Lee, H.K.; Kim, H.C.; Hna, J.H.; Lee, K.B.; Lim, E.H.; Shin, S.H.; Jin, H.A.; Cho, E.G.; Kim, Y.M.; Yoo, C. Analysis of the National Air Pollutant Emission Inventory (CAPSS 2016) and the Major Cause of Change in Republic of Korea. Asian Journal of Atmospheric Environment. 2020. 14(4), 422-445.

19. Bae, S.H.; Kwon, H.J. Current State of Research on the Risk of Morbidity and Mortality Associated with Air Pollution in Korea. Yonsei Medical Journal. 2019, 60(3), https://eymj.org/DOIx.php?id=10.3349/ymj.2019.60.3.243

20. Tran, H.; Kim J.y.; Kim, D.U.; Choi, M.Y.; Choi, M.H. Impact of air pollution on cause-specific mortality in Korea: Results from Bayesian Model Averaging and Principle Component Regression approaches. Science of the Total Environment. 2018. 636. 10201031. https://doi.org/10.1016/j.scitotenv.2018.04.273

21. Anenberg, S.C.; Balakrishnan, K.; Jetter, J.; Masera, O.; Mehta, S., Moss, J.; Ramanathan, V. Cleaner Cooking Solutions to Achieve Health, Climate, and Economic Cobenefits. Environmental Science \& Technology. 2013. 47(9), $3944-3952$. https://doi.org/10.1021/es304942e

22. Kapadia, Z.Z.; Spracklen, D.V.; Arnold, S.R.; Borman, D.J.; Mann, G.W.; Pringle, K.J.; Monks, S.A.; Reddington, C.L.; Benduhn, F.; Rap, A.; Scott, C.E.; Butt, E.W.; Yoshioka, M. Impacts of aviation fuel sulfur content on climate and human health. Atmospheric Chemistry and Physics. 2016. 16(16), 10521-10541. https://doi.org/10.5194/acp-16-10521-2016.

23. Kodros, J.K.; Scott, C.E.; Farina, S.C.; Lee, Y.H.; L'Orange, C.; Volckens, J.; Pierce, J.R. Uncertainties in global aerosols and climate effects due to biofuel emissions. Atmospheric Chemistry and Physics. 2015.15 (15), 8577-8596. https://doi.org/10.5194/acp-158577-2015.

24. Lang, J.L.; Zhou, Y.; Chen, D.S.; Xing, X.F.; Wei, L.; Wang, X.T.; Zhao, N.; Zhang, Y.Y.; Guo, X.R.; Han, L.H.; Cheng, S.Y. Investigating the contribution of shipping emissions to atmospheric PM2.5 using a combined source apportionment approach Environmental Pollution. 2017. 229, 557-566. http://dx.doi.org/10.1016/j.envpol.2017.06.087.

25. Wu, Y.Y.; Gu, B.J.; Erisman, J.W.; Reis, S.; Fang, Y.Y.; Lu, X.H.; Zhang, X.M. PM2.5 pollution is substantially affected by ammonia emissions in China. Environmental Pollution. 2016. 218, 86-94. http://dx.doi.org/10.1016/j.envpol.2016.08.027.

26. NIER. A Handbook of Method for National Air Pollutant Emission Estimation, National Institute of Environmental Research, Sejong, Korea, 2013

27. RDA. 2011 Survey on the Utilization of Agricultural Machinery and Farmwork Mechanization Rate, Rural Development Administration, Jeonju, Korea, 2012 